\section{HER2 splice variants and their relevance in breast cancer}

\author{
Marianna Sasso, Francesca Bianchi, \\ Valentina Ciravolo, Elda Tagliabue, \\ Manuela Campiglio
}

Molecular Targeting Unit, Department of Experimental Oncology, Fondazione IRCCS Istituto Nazionale dei Tumori, Milan, Italy

\section{Abstract}

The HER2 gene amplification occurs in 20 $30 \%$ of breast cancer and is correlated with a poorer prognosis compared to HER2-negative disease due to increased proliferation and metastatic potential. Two major types of receptor inhibitors have been developed for therapy and one for each categories is currently used in clinic: i) the humanized monoclonal antibody trastuzumab, directed against the HER2 extracellular domain; and ii) the EGFR/HER2 dual tyrosine kinase inhibitor lapatinib. However, patients may develop resistance to drugs and show disease progression. Several mechanism of resistance have been explored and are still under investigation. Here, we focus our attention on the role played by the alternative splicing forms of HER2 in mediating HER2 oncogenic activity and in conditioning the response to HER2 therapies. Three HER2 splice variants have been described so far; the p100 and the herstatin give raise to two secreted proteins of $100 \mathrm{kd}$ and $68 \mathrm{kd}$, respectively, that act as cell growth inhibitors. The third splice form of HER2 gene is the $\triangle 16$ HER2, encoding for a receptor lacking exon16, whose absence determines constitutive active dimers with transforming activity in vitro and in vivo. The $\triangle 16 \mathrm{HER} 2$ binds to trastuzumab to a less extend, due to conformational changes of the extracellular domain and its levels are supposed to increase proportionally to the increasing of the HER2 wild-type copy numbers in human primary breast cancers. Finally, HER2 carboxy-terminal fragments (CTFs), generated by alternative initiation of translation, were observed in breast cancer patients. In particular, 611-CTF, activating multiple signaling pathways since it is expressed as a constitutively active homodimer, was suggested to be a potent oncogene capable of promoting mammary tumour progression and metastasis. Despite the evidences of a potential role of the naturally occurring inhibitors p100 and herstatin on the wild-type HER2 and its signaling pathway, to date they do not seem to have a possible clinical development. To date the most promising forms currently under investigation that could have a key role in determining the increased HER2-positive tumours aggressiveness and toward the development of bio-drugs are the HER $2 \Delta 16$ and the CTFs.

\section{HER2 features and relevance in breast cancer disease}

HER2 (also know as c-HER-2 or HER2/neu) is a proto-oncogene mapped to the chromosome $17 q 21,{ }^{1,2}$ encoding a 1255 amino acid transmembrane glycoprotein of $185 \mathrm{kDa}^{3}$ designated as HER2 or p185 ${ }^{\text {HER2 }}$ (Figure 1A) that, together with its relatives HER1, HER3 and HER4, belongs to HER family of receptor tyrosine kinases (RTKs). ${ }^{4}$

The HER receptors share a similar structure, comprising an extracellular binding domain, a transmembrane lipophilic segment, and except for HER3, a functional intracellular tyrosine kinase domain with a regulatory carboxy-terminal tail. These RTKs are activated by the binding of specific EGF-like growth factors, ${ }^{5}$ but none of them directly binds to HER2. Ligands binding induce receptor homo- and hetero-dimerization and tyrosine autophoshorylation, which are obligatory steps in signal activation. ${ }^{6}$ Despite orphan of a specific soluble ligand, HER2 is the preferred heterodimerization partner of the other three HER members, ${ }^{7}$ since it adopts a fixed conformation resembling a ligand-activated state, allowing it to dimerize in the absence of ligand. ${ }^{8}$ Once activated, it is able to induce signaling that promotes proliferation and survival. ${ }^{9-12}$ HER2 amplification occur in $20-25 \%$ of breast cancers leading to poor prognosis. ${ }^{13-16}$ Indeed, HER2 amplification leads to progression from normal breast epithelia to invasive cancer cells. ${ }^{17-19}$ Accordingly, gene expression analysis showed that the HER2-positive tumours clusterized as a specific subset, mainly characterized by the lack of expression of genes associated with hormone receptor signaling pathways and high-level expression of a cluster of genes associated with proliferation. This subgroup was clearly distinguished from the other aggressive phenotype (to which triple negative breast cancer mainly belongs), defined by lacking of HER2 expression and both estrogen and progesterone receptors, from luminal, expressing estrogen receptor, and normal-like subset. ${ }^{20}$ However, the HER2 overexpression is necessary but not sufficient to induce malignant transformation, as clearly demonstrated by rat proto-oncogene HER2/neu transgenic mice either bearing additional alterations in HER2 gene sequence ${ }^{21,22}$ or cross-breeded with mice transgenically expressing other cancerrelated alterations. ${ }^{23-25}$
Correspondence: Manuela Campiglio, Molecular Targeting Unit, Dept. of Experimental Oncology, Fondazione IRCCS Istituto Nazionale dei Tumouri, via Venezian 1, 20133 Milan, Italy. Tel. +390223905121 - Fax. +390223902692.

E-mail: manuela.campiglio@istitutotumori.mi.it

Key words: HER2, breast cancer, HER2delta16, Herstatin, ECD.

Conflict of interest: the authors report no conflicts of interest.

Received for publication: 4 June 2011.

Revision received: 7 June 2011.

Accepted for publication: 7 June 2011.

This work is licensed under a Creative Commons Attribution NonCommercial 3.0 License (CC BYNC 3.0).

(C) Copyright M. Sasso et al., 2011

Licensee PAGEPress, Italy

Journal of Nucleic Acids Investigation 2011; 2:e9 doi:10.4081/jnai.2011.e9

The increased HER2 expression levels in breast carcinoma respect to normal breast tissue and the evidence of its driving role in HER2-positive tumours progression made HER2 an ideal target for specific therapeutic approaches. Indeed, in 1998 trastuzumab, a recombinant humanized monoclonal antibody directed to the extracellular domain of the HER2 protein, was the first monoclonal antibody to gain the FDA approval for clinical treatment of women with HER2 positive metastatic breast cancer. ${ }^{26}$ Since 2006 trastuzumab was also approved for adjuvant treatment of patients with early breast cancer and promising evidences showed its effect also in preoperative setting. ${ }^{27,28}$ The other HER2-targeted therapy approved for clinical use is the EGFR/HER2 dual tyrosine kinase inhibitor lapatinib, a competitor of ATP in the kinase domain of HER2 that impairs the transmission of its signal. ${ }^{29,30}$ Based on promising clinical trials data, ${ }^{31,34}$ in 2007 FDA and EMEA approved the use of lapatinib in combination with capacitabine in patients with advanced or metastatic HER2-positive breast cancers. ${ }^{35}$ Despite objective clinical results, obtained with biodrugs so far anti-HER2 agents show clinical benefit in about 50\% of patients with HER2positive breast carcinomas. ${ }^{36,39}$ Extensive literature aimed to clarify the mechanisms of trastuzumab efficacy and resistance in different breast cancer clinical settings has been published so far, ${ }^{40}$ and a huge effort has been devoted to the search for markers of response to therapy. In spite of high investments, none of the markers described as associated to therapy sensitivity/resistance were sufficiently 
reliable to be introduced into the clinical setting.

It is now clear the importance of a persistent targeting of HER2 axis in breast cancer and, therefore, to develop additional therapeutic strategies to better impair HER2 activity. This will be reached through a more precise delineation of both HER2 biology and HER2 drugs mechanisms of action in tumours, ${ }^{44}$ especially after the recent demonstration of improved overall survival of gastric patients bearing HER2 amplification and, therefore, trastuzumab-treated in phase III trial. Indeed, it is unlikely that the optimization of both treatment protocols design and duration will be sufficient to overcome HER2 resistance. ${ }^{40}$ It is, therefore, necessary to come back to dissect HER2 pathway and unravel key features contributing to its transforming capacity. In the present review, we focus our attention on the role played by the alternative splicing forms of HER2 in mediating HER2 oncogenic activity and in conditioning the response to HER2 therapies (summarized in Table1), in breast cancer.

\section{p100: ECD from the splicing processing}

The first described HER2 splice variant was the extracellular secreted $100 \mathrm{kDa}$ fragments named p100. In 1993 Scott et al. ${ }^{41}$ described a $2.3 \mathrm{~kb}$ variant of HER2 mRNA encoding the first 633 amino acids (aa) containing almost the entire HER2-ECD (extra cellular domain) (subdomains I-IV) (Figure 1B). Many transmembrane growth factor receptors have been reported to have soluble, ligand-binding receptor forms detectable in the conditioned media of tumour cells and in biological fluids. These soluble receptor proteins arise through proteolytic cleavage of membrane-anchored fulllength receptors ${ }^{42}$ and/or by alternative splicing or other gene rearrangements that usually produce novel transcripts which encode for proteins containing parts of the ECD but lacking the transmembrane and cytoplasmic domains of the full-length receptor. ${ }^{43}$

p100 has been described to interfere with oncogenic HER2 activity through different mechanisms. Collectively, it acts as inhibitor of tumour cell proliferation ${ }^{41,44-46}$ (Table 1). Aigner et al. showed that this 100 kDa HER2ECD can act as a dominant-negative inhibitor of growth factor-mediated tumour cell proliferation. ${ }^{47}$ They transfected MCF7 breast cancer cells, since MCF7 does not endogenously express this spliced HER2 mRNA but only low levels of the full-length HER2 protein. Doxycycline-regulated expression of the transfected HER2-ECD cDNA induced the HER2-

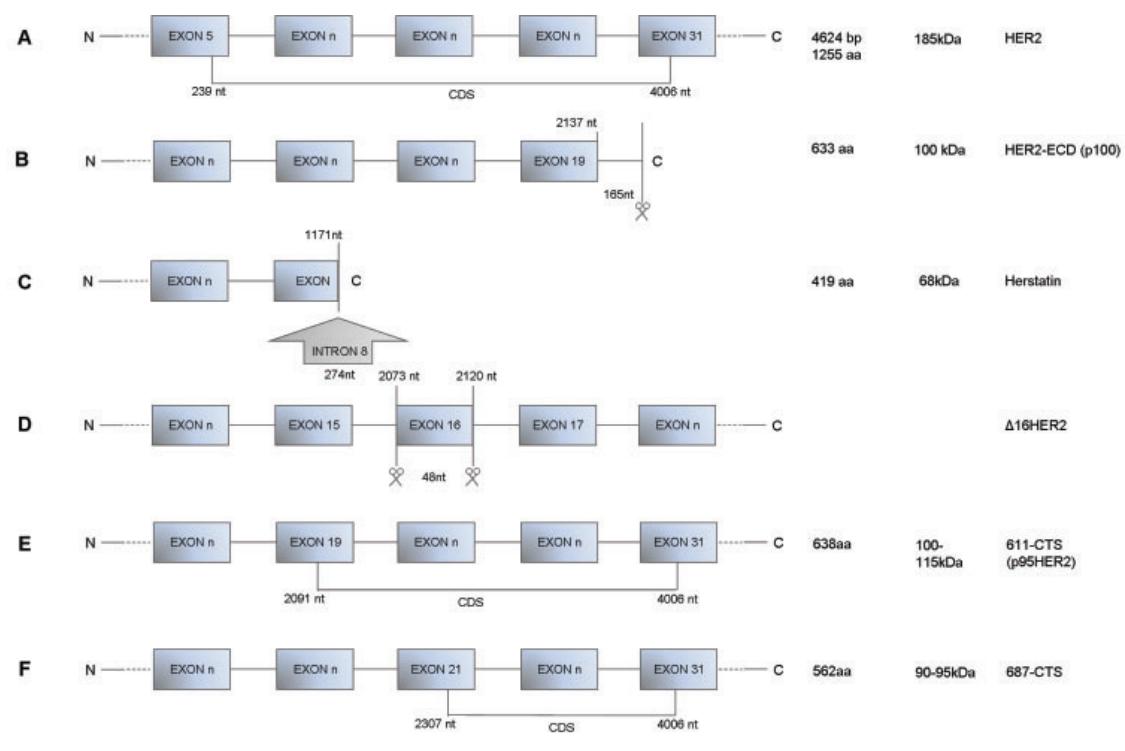

Figure 1. Structures of HER2 transcript variants. A) Full-length HER2; NCBI Reference Sequence: NM_004448.2. The AUG start codon is located at position 239 and the coding sequence $(\mathrm{CDS})$ ends at position 4006. B) HER2-ECD (p100); the $3^{\prime}$ end of the truncated transcript reveals an exonic extension of $165 \mathrm{bp}$ with an in-frame stop codon and a poly(A) addition site. C) Herstatin; the amino acid sequence is identical to the full-length HER2 mRNA until amino acid residue 340. A 274-nt insertion located between nucleotide residues 1171 and 1172 of the full-length mRNA results in frame with HER2 exon sequence and encodes a 79-aa extension after amino acid residue 340. D) $\triangle 16 H E R 2$; entire exon 16, from nucleotide 2073 to nucleotide 2120, is skipped from full-length HER2 mRNA. E) 611-CTS; the 100- to 115-kDa p95HER2 fragment generated by alternative initiation of translation from the AUG codon in position 611. F) 687-CTS; the 90to $95-\mathrm{kDa}$ fragment generated by alternative initiation of translation from the AUG codon in position 687 . The $\mathrm{N}$ at the beginning of each rectangles series representing an HER2 transcript identifies the amino terminus; the $\mathrm{C}$ at the end identifies the carboxy terminus.

ECD-mediated inhibition of spontaneous proliferation as well as inhibition of heregulinmediated proliferation and signal transduction. As expected, significant amounts of p100 protein in the conditioned media has been detected, demonstrating that the HER2-ECD protein was effectively secreted in transfected MCF7 cells. Likewise, in MKN7, a gastric tumour cell line with very high expression levels of the splice variant as well as full-length HER2 proteins, ribozyme-targeting of the endogenously expressed p100 mRNA splice variant demonstrated reduction of p100-mediated inhibition of proliferation in soft agar accordingly with a reduced downstream signaltransduction. Indeed, p100 over-expression resulted in a time-delay and decrease of heregulin-mediated phosphorylation of the HER4 receptor, followed by a similar inhibition of downstream signaling events such as activation of p44/p42 MAP-kinases.

Actually, several evidences point to HER2ECD levels in serum as biomarker for HER2 over-expressing cancer aggressiveness and therapy response. Indeed, HER2-ECD is easily detectable using an enzyme-linked immunosorbent assay (ELISA). ${ }^{48}$ The potential in vivo role of soluble truncated HER2 proteins was investigated in gastric tumours, where a trend was found towards reduced HER2-ECD expression in tumours with a more aggressive phenotype. $^{49}$ Clearly, the great part of data came from breast cancer studies where serum ECD levels have been proposed as predictive marker for trastuzumab treatment. Several studies have been performed providing contrasting conclusions, therefore, the utility of serum ECD values at baseline and during therapy as a potential marker of tumour response or progression is actually a matter of debate. ${ }^{49-55}$

\section{Herstatin: a naturally occurring HER2 inhibitor}

In 1999 Doherty et al. ${ }^{44}$ described a secreted protein of $68 \mathrm{kDa}$, named herstatin, as the product of an alternative HER2 transcript which is generated by retention of intron 8 in HER2 alternative mRNA (Figure 1C). This transcript generates a protein that consists of the first 340 aa-residues identical to N-terminal subdomains of HER2, followed by a novel Cterminus of 79 aa-residues. Herstatin appears 
to be a naturally occurring inhibitor of HER-2, because it disrupts dimers, reduces tyrosine phosphorylation of HER2, and inhibits the anchorage-independent growth of transformed cells that overexpressed HER2. Azios et al. underlined the importance of herstatin in cancer disease since its ectopic expression leads not only to the interruption of constitutive activation of HER2, but also to the lack of heterodimerization of HER2/HER3 and EGFR activation. The ability of herstatin to suppress colony formation of HER2 or EGFR-overexpressing cells suggested its potential use in limiting tumour cell growth driven by these receptors. ${ }^{45}$ In $2005 \mathrm{Hu}$ et al. described the three dimensional structure of herstatin and its interaction binding site with HER2 extracellular domain. ${ }^{56}$ The interaction of herstatin and HER2 on the cell surface was accompanied by an increased co-localization of HER2 and herstatin in the cytoplasm, suggesting that HER2/herstatin complex formation may prevent transit from endoplasmic reticular to cell surface of HER2. The intracellular sequestering of HER2 by herstatin may be a possible naturally occurring inhibitory mechanism controlling cell growth ${ }^{57}$ (Table 1).

Several studies investigated the presence of herstatin in normal and cancer cellular lines. Herstatin mRNA was found expressed in normal human fetal kidney and liver tissue ${ }^{58}$ but it appears to be expressed at reduced levels respect to the HER2 mRNA in breast carcinoma cells that contain amplification of HER2. ${ }^{59}$ A further study investigated the herstatin mRNA level and its protein expression in breast carcinoma tissues compared to their normal breast tissues. In this context, it has been shown that herstatin mRNA and protein are expressed in non-cancerous breast, in areas adjacent to breast carcinoma. This characteristic expression pattern of herstatin in non-cancerous breast tissues might contribute to the normally effective endogenous HERsinhibition system, because there is a local need for HERs inhibition in differentiating ductal ephitelia.$^{60}$ An additional study showed that breast cancer tissues express Herstatin mRNA, but the protein is absent in $75 \%$ of breast carcinomas, which indicates that cancer cells are protected by not yet established intrinsic mechanisms against the putative growth-inhibitory effects of this molecule. The authors speculate that, if this negative regulation occurs at the pre-translational level, then it seems possible that exogenous administration of i.e pan-HER antibodies would help in blocking tumour growth. If the protein is produced but released from the cells, then it seems possible that the mechanism, which impairs the endogenous inhibitor activity, will also apply to the exogenous administered antibody as well. Most importantly, if $25 \%$ of breast

Table 1. Different HER2 isoforms with their biological activities and target therapies.

\begin{tabular}{|c|c|c|c|}
\hline \multicolumn{2}{|c|}{ HER2 isoforms } & Biological activity & Targeted therapies used \\
\hline \multicolumn{2}{|l|}{ p100 } & Inhibition of tumour cells proliferation & Contrasting data on trastuzumab \\
\hline \multicolumn{2}{|c|}{ Herstatin } & Inhibition of tumour cells proliferation & \\
\hline \multicolumn{2}{|c|}{$\triangle 16$ HER2 } & $\begin{array}{l}\text { Oncogenic capability; increase of metastatic } \\
\text { potential }\end{array}$ & $\begin{array}{l}\text { Lower trastuzumab efficacy; } \\
\text { de novo tamoxifen resistance }\end{array}$ \\
\hline CTFs & $\begin{array}{l}\text { 611-CTF } \\
\text { 687-CTF } \\
648-\mathrm{CTF}\end{array}$ & $\begin{array}{l}\text { Increase of metastatic potential } \\
\text { lapatinib response } \\
\text { Inactive } \\
\text { The same of full-lenght HER2 }\end{array}$ & Trastuzumab resistance \\
\hline
\end{tabular}

carcinomas grow in the presence of an endogenous pertuzumab-like inhibitor, which is produced in high amounts in some cases with adverse prognostic parameters (HER2 overexpression, activated Akt/PKB, and blocked p21CIP1/WAF1), then it remains questionable whether these cancers would benefit from exogenous attempts to disrupt HER2 dimerization. ${ }^{61}$ It has been shown that Herstatin expression inhibited the in vitro growth of the human glioblastoma cell line U87MG in a dose responsive manner and that prevented the tumours formation. ${ }^{46}$ Additionally, authors demonstrated that human glioblastoma bearing truncated EGFR were resistant to Herstatin, suggesting that herstatin may have utility against glioblastoma driven by the EGFR. $^{46}$

\section{$\triangle 16 H E R 2:$ the real player in HER2 tumourigenesis?}

An important issue concerning HER2 overexpression in human primary breast cancers is that this genetic alteration, primarily due to gene amplification, is relevant but not sufficient to induce transformation. The expression of an alternatively spliced human HER2 isoform encoding a receptor lacking exon 16, which immediately precedes the transmembrane domain, and so called $\triangle 16$ HER2 (Figure 1D). It was reported this splice variant accounts only for $4-9 \%$ of the total HER2 transcripts, but transgenic studies provided direct evidence of the key role of the $\triangle 16$ HER 2 splice variant in HER2 transforming activity. In transgenic mice, tumours arose only when the oncoprotein carried small deletions in the extracellular domain, thus promoting HER2/neu transforming activity through formation of intermolecular disulfide bonds. ${ }^{21}$ Indeed, mammary-specific expression of the rat HER2/neu gene induces tumours only when accompanied by in-frame activating deletions of cysteine residues within the wild-type HER2/neu extracellular domain. ${ }^{62}$ The loss of these cysteine residues appears to induce a conformational change in the HER2 extracellular domain that promotes intermolecular disulfide bridges and, in turn, constitutively activates stable HER2 homodimers on tumour cell surface able to drive mitogenic signaling. ${ }^{63}$ Athymic mice injected with $\triangle 16$ HER2-HEK293 transfectants developed tumours, whereas mice injected with HEK293 control cells ectopically overexpressing only WT HER2, did not. ${ }^{64}$ In this model, $\triangle 16 \mathrm{HER} 2$ was constitutively active supporting the hypothesis that its tumourigenic potential is due to the ability to form disulfide-bridged homodimers. More recently, Mitra et $a l .{ }^{65}$ confirmed the same behavior in a breast cancer model. Indeed, they described stable dimers in MCF7 cells transfected with $\triangle 16$ HER2. As expected, ectopic $\triangle 16 H E R 2$ expression led to increased activation of multiple oncogenic pathways, e.g., FAK, Src kinase, phosphatidylinositol 3-kinase/AKT, and mitogen-activated protein kinase, as compared to cells expressing WT HER2. The activation of these oncogenic cascades results in a dramatic increased of $\triangle 16$ HER2-MCF7 migration and invasion. The most interesting data were the positive correlation between $\triangle 16 H E R 2$ expression and positive lymph nodes in patients with HER2-positive tumours highlighting $\triangle 16 \mathrm{HER} 2$ as a critical feature for HER2-breast cancer progression. This study also anticipated the potential clinical implications of $\triangle 16 H E R 2$ variant expression in antiHER2 targeted drugs susceptibility. Castiglioni et $a l^{64}$ demonstrated the lower reactivity of trastuzumab, in comparison with other antiHER2/ECD Mabs binding to different HER2/ECD epitopes. These finding suggested that $\triangle 16$ HER2 expression could be relevant for HER2-targeted therapies efficacy. This hypothesis is consistent with clinical findings indicating that wild-type HER2 gene amplification in human primary breast cancers determined a proportional increase in $\triangle 16 \mathrm{HER} 2$ levels. ${ }^{66}$ Therefore, we can speculate that decreased susceptibility to trastuzumab observed in breast cancer patients with HER2 FISH ratios $>8^{67}$ (Table 1) can be attribute to their high expression level of $\triangle 16 H E R 2$. Transformation associated with HER2 overexpression might 
reflect the increase in absolute levels of this splice variant to a critical threshold for constitutive activation of HER2.

Besides the resistance to anti-HER2 therapy, $\triangle 16$ HER2 was described as involved in endocrine resistance in HER2-positive/ER-positive breast carcinomas (Table 1). These tumours, that account for half of HER2 positive tumours, represent a challenge for the oncological treatment, since more than $70 \%$ exhibited de novo tamoxifen resistance ${ }^{68}$ and, indeed, continue to growth even after estrogen depletion. ${ }^{69}$ Preclinical studies demonstrated that $\triangle 16$ HER2, but not wild-type HER2, promotes estrogen-independent growth and the novo resistance to tamoxifen treatment therapy in breast cancer cells in vivo and in vitro. To date, two mechanisms can explain the acquisition of hormone resistance in breast cancer mediated by HER $2 \Delta 16$. In the first, HER $2 \Delta 16$ transfected breast cancer tamoxifen-treated cells were found to up-regulate BCL-2 expression mainly through the suppression of miR15a/16 compared with tamoxifen-sensitive cell lines. Normally, BCL-2 translation is repressed by binding of miR-15a or miR-16 to a seed sequence in BCL-2 mRNA 3 '-untranslated regions, and loss of miR-15a/16 in several cancer cell lines and tumours is associated with BCL-2 upregulation ${ }^{69-71}$ and resistance to therapy. ${ }^{71}$ Consistently, BCL-2 is upregulated in HER2 $\Delta 16$ expressing tamoxifen-resistant cells where levels of miR-15a/16 were reduced. Authors established an in vivo model that revealed the inability of wild-type HER2 preclinical models to fully recapitulate the aggressive and variable clinical nature of HER2-positive breast tumours. Indeed, similarly to clinical observations, HER2 $\Delta 16$-expressing xenografts are both tamoxifen resistant and estrogen independent, whereas consistent with other reports, ${ }^{70}$ HER2-expressing xenografts display only partial acquired tamoxifen resistance and remain estrogen-dependent. The second mechanism is based on the down-regulation of mir-342 observed in breast cancer cells expressing HER $2 \Delta 16$ and in primary breast tumours of patients who failed tamoxifen therapy. Mir-342 controlled expression of genes involved in tamoxifen mediated response in breast cancer cells and, indeed, its restoration in MCF7/ HER2 $\Delta 16$ sensitized these cells to tamoxifen-induced apoptosis with a dramatic reduction in cell growth. ${ }^{70}$

HER2 $\Delta 16$ was definitely demonstrated to be sufficient per se for mammary tumours development as we recent reported. ${ }^{63}$ We established the first mouse line that transgenically expresses both human $\triangle 16 \mathrm{HER} 2$ and firefly luciferase genes. A higher tumour incidence, a more rapid tumour growth, as well as a significantly shorter latency period (15.11 vs 28.6 weeks) in $\triangle 16$ HER2-LUC transgenic mice were observed, as compared with MMTVhuHER2, the other mice model transgenic for the human wild-type HER2. ${ }^{72}$ The signaling activity of over expressed $\triangle 16$ HER2LUC revealed that the oncogenic properties of $\triangle 16 H E R 2$ were mediated through activation of Src kinase. ${ }^{63}$

Notably, only 5 copies were found to be sufficient to drive neoplastic transformation of mammary epithelial cells in $\triangle 16$ HER2-LUC mice, whereas 30-50 wtHER2 transgene copies are required to induce breast cancer in about $80 \%$ of MMTV-wtHER2 transgenic mice. ${ }^{73}$

All these findings demonstrated that $\triangle 16 H E R 2$ splice variant could actually represent the transforming form of HER2 oncoprotein. Therefore, the role of HER2 $\Delta 16$ in HER2driven breast tumours progression and therapy response could be underestimated. There is the need to develop screening test for HER2 $\Delta 16$ expression in cancer tissues but, most of all, to find compounds able to interfere with its binding to HER2 receptor, to try to overcome the HER $2 \Delta 16$ induced resistance.

\section{Carboxy-terminal fragments: besides HER2 splicing processing}

A subgroup of HER2-positive patients expresses a series of carboxyl-terminal fragments (CTFs) of HER2. ${ }^{74}$ HER2 CTFs can be generated at least by two independent mechanisms: proteolytic shedding and alternative initiation of translation. In the first scenario, metalloproteases shed the extracellular domain of HER2 at a site proximal to the transmembrane domain, generating a 95- to 100 kDa fragment, known as p95HER2, that starts at alanine $648 .{ }^{74-75}$ Alternative initiation of translation of the mRNA encoding HER2 from 2 internal initiation methionine at positions 611 and 687 (codons numbered according to the full-length molecule) generates two additional p95HER2 fragments of 100 to $115 \mathrm{kDa}$ and $90-$ to $95-\mathrm{kDa}$, respectively known as 611 CTF (Figure 1E) and 687-CTF (Figure 1F). They differ in a stretch of 76 amino acids, which includes the transmembrane domain and a cysteine-rich short extracellular domain. ${ }^{73}$ Even though lacking a signal peptide, the 611-CTF fragment is efficiently incorporated into the secretory pathway and transported to the plasma membrane. Instead, the 687-CTF fragment can be found both in the cytoplasm and nucleus. ${ }^{76}$

Pedersen and colleagues analyzed the activity of the individual p95HER2 (Table 1). They showed that the soluble intracellular 687-CTF fragment, despite having an intact kinase domain, was inactive. In contrast, the two CTFs containing the transmembrane domain,
648- and 611-CTFs, can activate several intracellular signal transduction pathways. ${ }^{76}$ Interestingly, the level of activation of these pathways is quite different between the two HER2 CTFs. 611-CTF activates the mitogenactivated protein kinase and the Akt pathways to a greater extent respect to 648-CTF, because it constitutively forms homodimers maintained through disulfide bonds. In contrast, 648-CTF does not seem to form homodimers, and its activity is comparable with that of fulllength HER2. ${ }^{76}$ As a result, expression of the 611-CTF fragment leads to the regulation of a specific set of genes. Several of these genes, such as MMP1, ANGPTL4, MET, CD44, PLAUR, EPHA2, ITGA2, ITGFB, TGFA, and IL-11, are causally involved in the metastatic progression. ${ }^{76}$ Moreover, cortactin, a cytoskeletonbinding protein involved in the regulation of cell migration, was identified as a phosphoprotein regulated by 611-CTF. The authors showed that expression of 611-CTF leads to an increase in the phosphorylation of cortactin and, at least, to an increase in breast cancer cells motility (Table 1). ${ }^{77}$

According with all this evidence, it has been shown that breast cancer patients expressing CTFs have worse prognosis and are more likely to develop nodal metastasis compared with patients expressing predominantly full-length HER2 ${ }^{78}$ An early study by Christianson and colleagues showed that the expression of p95HER2 (611-CTF; 648-CTF) in breast tumours correlated with metastasis to the lymph nodes. ${ }^{73}$ Several subsequent studies supported that p95HER2 may be used as a biomarker of an aggressive subtype of HER2-positive breast cancer. ${ }^{78,79}$ Retrospective studies showed that tumours expressing p95HER2 tend to be resistant to treatment with trastuzumab ${ }^{80,81}$ but do respond to lapatinib. ${ }^{82}$ The effectiveness of lapatinib on p95HER2postive tumours is not surprising because the tyrosine kinase inhibitor also blocks the activity of the p95HER2 fragments. ${ }^{76}$ Therefore, tyrosine kinase inhibitors may be a good therapeutic approach to treat p95HER2-positive tumours. Because both the 95 - to $100-\mathrm{kDa}$ and the 100- to 115 -kDa transmembrane p95HER2 fragments lack the epitope recognized by trastuzumab, an obvious explanation for the lack of response to the antibody in p95HER2positive tumours is that expression of these fragments drives tumour growth even under treatment with trastuzumab.

\section{Conclusion and perspective}

Despite the evidence of a potential role of the naturally occurring inhibitors p100 and herstatin on the wild-type HER2 and its signal- 
ing pathway, to date they do not seem to have a possible clinical application. The most promising forms under investigation for a key role in determining the increased HER2-positive tumours aggressiveness and toward development of biodrugs are the HER $2 \Delta 16$ and the CTFs. Specific assays to determine and quantify the expression level of HER $2 \Delta 16$ have been already developed ${ }^{64}$ and the availability of transgenic mice models expressing these variants in the mammary gland will allowed to dissect the oncogenic mechanism of these forms, as well as to design new therapeutic molecules able to inhibits HER2 $\Delta 16$ dimers resistant to the HER2 targeted drug, such as trastuzumab.

\section{References}

1. Coussens L, Yang-Feng TL, Liao Y-C, et al. Tyrosine kinase receptor with extensive homology to EGF receptor shares chromosomal location with neu oncogene. Science 1985;230:1132-9.

2. King CR, Kraus MH, Aaronson SA. Amplification of a novel v-erb-B related gene in a human mammary carcinoma. Science 1985;229:974-6.

3. Akiyama T, Kadooka T, Ogawara H, Sakakibara S. Characterization of the epidermal growth factor receptor and the erbB oncogene product by site-specific antibodies. Arch Biochem Biophys 1986;245:531-6.

4. Hung M-C, Schechter AL, Chevray PY, et al. Molecular cloning of the neu gene: absence of gross structural alteration in oncogenic alleles. Proc Natl Acad Sci USA 1986;83:261-4.

5. Beerli RR, Hynes NE. Epidermal growth factor-related peptides activate distinct subsets of ErbB receptors and differ in their biological activities. J Biol Chem 1996;271:6071-6.

6. Heldin CH. Dimerization of cell surface receptors in signal transduction. Cell 1995;80:213-23.

7. Graus-Porta D, Beerli RR, Daly JM, Hynes NE. ErbB-2, the preferred heterodimerization partner of all ErbB receptor, is a mediator of lateral signaling. EMBO J 1997;16:1647-55.

8. Cho HS, Mason K, Ramyar KX, et al. Structure of the extracellular region of HER2 alone and in complex with the Herceptin Fab. Nature 2003;421:756-60.

9. Yarden Y, Sliwkowski MX. Untangling the ErbB signalling network. Nat Rev Mol Cell Biol 2001;2:127-37.

10. Olayioye MA, Neve RM, Lane HA, Hynes NE. The ErbB signaling network: receptor heterodimerization in development and cancer. EMBO J 2000;19:3159-67.
11. Citri A, Yarden Y. EGF-ERBB signalling: towards the systems level. Nat Rev Mol Cell Biol 2006;7:505-16.

12. Schlessinger J. Common and distinct elements in cellular signaling via EGF and FGF receptors. Science 2004;306:1506-7.

13. Ménard S, Pupa SM, Campiglio M, Tagliabue E. Biologic and therapeutic role of HER2 in cancer. Oncogene 2003;22:6570-8.

14. Slamon DJ, Clark GM, Wong SG, et al. Human breast cancer: correlation of relapse and survival with amplification of the HER-2/neu oncogene. Science 1987;235:177-82.

15. Hynes NE, Stern DF. The biology of erbB2/neu/HER-2 and its role in cancer. Biochim Biophys Acta Rev Cancer 1994;1198:165-84.

16. Holbro T, Hynes NE. ErbB receptors: directing key signaling networks throughout life. Annu Rev Pharmacol Toxicol 2004;44:195-217.:195-217.

17. Ménard S, Forti S, Castiglioni $F$, et al. HER2 as a prognostic factor in breast cancer. Oncology 2001;61:67-72.

18. Masood S, Bui MM. Prognostic and predictive value of HER2/neu oncogene in breast cancer. Microsc Res Tech 2002;59:102-8.

19. Ménard S, Tagliabue E, Campiglio M, Pupa SM. Role of HER2 gene overexpression in breast carcinoma. J Cell Physiol 2000;182:150-62.

20. Sorlie T, Perou CM, Tibshirani R, et al. Gene expression patterns of breast carcinomas distinguish tumour subclasses with clinical implications. Proc Natl Acad Sci U S A 2001;98:10869-74.

21. Siegel PM, Ryan ED, Cardiff RD, Muller WJ. Elevated expression of activated forms of Neu/ErbB-2 and Erb-3 are involved in the induction of mammary tumours in transgenic mice: implications for human breast cancer. EMBO J 1999;18:2149-64.

22. Chan R, Muller WJ, Siegel PM. Oncogenic activating mutations in the neu/erbB-2 oncogene are involved in the induction of mammary tumours. Ann N Y Acad Sci 1999; 889:45-51.

23. Muller WJ, Arteaga CL, Muthuswamy SK, et al. Synergistic interaction of the neu proto-oncogene product and transforming growth factor a in the mammary epithelium of transgenic mice. Mol Cell Biol 1996; 16:5726-36.

24. Li B, Rosen JM, Menamin-Balano J, et al. neu/ERBB2 cooperates with p53-172H during mammary tumourigenesis in transgenic mice. Mol Cell Biol 1997;17:3155-63.

25. Casalini P, Botta L, Ménard S. Role of p53 in HER2-induced proliferation or apoptosis. J Biol Chem 2001;276:12449-53.

26. Pegram MD, Lipton A, Hayes DF, et al. Phase II study of receptor-enhanced chemosensitivity using recombinant humanized anti-p185HER2/neu monoclonal antibody plus cisplatin in patients with HER2/neu-overexpressing metastatic breast cancer refractory to chemotherapy treatment. J Clin Oncol 1998;16:2659-71.

27. Gennari R, Ménard S, Fagnoni F, et al. Pilot study of the mechanism of action of preoperative trastuzumab in patients with primary operable breast tumours overexpressing HER2. Clin Cancer Res 2004;10: 5650-5.

28. Gianni L, Eiermann W, Semiglazov V, et al. Neoadjuvant chemotherapy with trastuzumab followed by adjuvant trastuzumab versus neoadjuvant chemotherapy alone, in patients with HER2-positive locally advanced breast cancer (the NOAH trial): a randomised controlled superiority trial with a parallel HER2-negative cohort. Lancet 2010;375: 377-84.

29. Spector NL, Xia W, Burris H, III, et al. Study of the biologic effects of lapatinib, a reversible inhibitor of ErbB1 and ErbB2 tyrosine kinases, on tumour growth and survival pathways in patients with advanced malignancies. J Clin Oncol 2005; 23:2502-12.

30. Lackey KE. Lessons from the drug discovery of lapatinib, a dual ErbB1/2 tyrosine kinase inhibitor. Curr Top Med Chem 2006;6:435-60.

31. Burstein HJ, Storniolo AM, Franco S, et al. A phase II study of lapatinib monotherapy in chemotherapy-refractory HER2-positive and HER2-negative advanced or metastatic breast cancer. Ann Oncol 2008;19:106874.

32. Blackwell KL, Burstein H, Pegram M, et al. Determining relevant biomarkers from tissue and serum that may predict response to single agent lapatinib in trastuzumab refractory metastatic breast cancer. J Clin Oncol 2005;23:256s-Abstract 3004.

33. Iwata H, Toi M, Fujiwara Y, et al. Phase II clinical study of lapatinib (GW572016) in patients with advanced or metastatic breast cancer. Breast Cancer Symphosium 2006;Abstract1091.

34. Gomez HL, Doval DC, Chavez MA, et al. Efficacy and safety of lapatinib as first-line therapy for ErbB2-amplified locally advanced or metastatic breast cancer. J Clin Oncol 2008;26:2999-3005.

35. Geyer CE, Forster J, Lindquist D, et al. Lapatinib plus capecitabine for HER2-positive advanced breast cancer. N Engl J Med 2006;355:2733-43.

36. Piccart-Gebhart MJ, Procter M, LeylandJones B, et al. Trastuzumab after adjuvant chemotherapy in HER2-positive breast cancer. N Engl J Med 2005;353:1659-72.

37. Romond EH, Perez EA, Bryant J, et al. 
Trastuzumab plus adjuvant chemotherapy for operable HER2-positive breast cancer. N Engl J Med 2005;353:1673-84.

38. Joensuu H, Kellokumpu-Lehtinen PL, Bono P, et al. Adjuvant docetaxel or vinorelbine with or without trastuzumab for breast cancer. N Engl J Med 2006;354: 809-20.

39. Campiglio M, Bufalino R, Sandri M, et al. Increased overall survival independent of RECIST response in metastatic breast cancer patients continuing trastuzumab treatment: evidence from a retrospective study. Breast Cancer Res Treat 2011;128:147-54.

40. Tagliabue E, Balsari A, Campiglio M, Pupa SM. HER2 as a target for breast cancer therapy. Expert Opin Biol Ther 2010;10: 711-24.

41. Scott GK, Robles R, Park JW, et al. A truncated intracellular HER2/neu receptor produced by alternative RNA processing affects growth of human carcinoma cells. Mol Cell Biol 1993;13:2247-57.

42. Pupa SM, Ménard S, Morelli D, et al. The extracellular domain of the c-erbB-2 oncoprotein is released from tumour cells by proteolytic cleavage. Oncogene 1993;8:2917-23.

43. Arribas J, Borroto A. Protein ectodomain shedding. Chem Rev 2002;102:4627-38.

44. Doherty JK, Bond C, Jardim A, et al. The HER-2/neu receptor tyrosine kinase gene encodes a secreted autoinhibitor. Proc Natl Acad Sci U S A 1999;96:10869-74.

45. Azios NG, Romero FJ, Denton MC, et al. Expression of herstatin, an autoinhibitor of HER-2/neu, inhibits transactivation of HER-3 by HER-2 and blocks EGF activation of the EGF receptor. Oncogene 2001;20:5199-209.

46. Staverosky JA, Muldoon LL, Guo S, et al. Herstatin, an autoinhibitor of the epidermal growth factor receptor family, blocks the intracranial growth of glioblastoma. Clin Cancer Res 2005;11:335-40.

47. Aigner A, Juhl H, Malerczyk C, et al. Expression of a truncated $100 \mathrm{kDa}$ HER2 splice variant acts as an endogenous inhibitor of tumour cell proliferation. Oncogene 2001;19:2101-11.

48. Payne RC, Allard JW, Anderson-Mauser L, et al. Automated assay for HER-2/neu in serum. Clin Chem 2000;46:175-82.

49. Lennon S, Barton C, Banken L, et al. Utility of serum HER2 extracellular domain assessment in clinical decision making: pooled analysis of four trials of trastuzumab in metastatic breast cancer. J Clin Oncol 2009;27:1685-93.

50. Ali SM, Carney WP, Esteva FJ, et al. Serum HER-2/neu and relative resistance to trastuzumab-based therapy in patients with metastatic breast cancer. Cancer 2008;113:1294-301.
51. Ali SM, Leitzel K, Lipton A, et al. Value of serum human epidermal growth factor receptor 2 (HER2)/neu testing for early prediction of response to HER2/neu-directed therapies is still an open one and deserves further study in large prospective trials. J Clin Oncol 2009;27:e273.

52. Leary AF, Hanna WM, van de Vijver MJ, et al. Value and limitations of measuring HER-2 extracellular domain in the serum of breast cancer patients. J Clin Oncol 2009;27:1694-705.

53. Tse C, Lamy PJ. Clinical utility of serum human epidermal growth factor receptor 2 Extracellular Domain Levels: Stop the Shilly-Shally--It Is Time for a WellDesigned, Large-Scale Prospective Study. J Clin Oncol 2009;27:e286-e287.

54. Ghedini GC, Ciravolo V, Tortoreto M, et al. Role of soluble HER2 extracellular domain in HER2-mediated tumour growth and in response to Trastuzumab. J Cell Physiol 2010;225:256-65.

55. Ciravolo V, Huber V, Ghedini GC, et al. Potential role of HER2-overexpressing exosomes in countering Trastuzumabbased therapy. J Cell Physiol 2011 Apr 4. doi: 10.1002/jcp.22773. [Epub ahead of print]

56. Hu P, Zhou T, Qian L, et al. Sequestering ErbB2 in endoplasmic reticulum by its autoinhibitor from translocation to cell surface: an autoinhibition mechanism of ErbB2 expression. Biochem Biophys Res Commun 2006;342:19-27.

57. Hu P, Feng J, Zhou T, et al. In vivo identification of the interaction site of ErbB2 extracellular domain with its autoinhibitor. J Cell Physiol 2005;205:335-43.

58. Cell Markers and Cytogenetics Committees College of American Pathologists. Clinical laboratory assays for HER-2/neu amplification and overexpression: quality assurance, standardization, and proficiency testing. Arch Pathol Lab Med 2002;126:803-8.

59. Ross JS, Fletcher JA. HER-2/neu (c-erbB2) gene and protein in breast cancer. Am J Clin Pathol 1999;112:S53-S67.

60. Jackson-Fisher AJ, Bellinger G, Breindel $\mathrm{JL}$, et al. ErbB3 is required for ductal morphogenesis in the mouse mammary gland. Breast Cancer Res 2008;10:R96.

61. Koletsa T, Kostopoulos I, Charalambous E, et al. A splice variant of HER2 corresponding to Herstatin is expressed in the noncancerous breast and in breast carcinomas. Neoplasia 2008;10:687-96.

62. Ursini-Siegel J, Schade B, Cardiff RD, Muller WJ. Insights from transgenic mouse models of ERBB2-induced breast cancer. Nat Rev Cancer 2007;7:389-97.

63. Marchini C, Gabrielli F, Iezzi M, et al. The human splice variant HER2delta16 induces rapid tumour onset in a reporter transgenic mouse. PLoS ONE 2011;29; 6:e18727.

64. Castiglioni F, Tagliabue E, Campiglio M, et al. Role of exon-16-deleted HER2 in breast carcinomas. Endocr Relat Cancer 2006;13:221-32.

65. Mitra D, Brumlik MJ, Okamgba SU, et al. An oncogenic isoform of HER2 associated with locally disseminated breast cancer and trastuzumab resistance. Mol Cancer Ther 2009;8:2152-62.

66. Mrhalova M, Kodet R. Paget's disease of the nipple: a copy number of the genes ERBB2 and CCND1 versus expression of the proteins ERBB-2 and cyclin D1. Neoplasma 2003;50:396-402.

67. Dowsett M, Procter M, Caskill-Stevens W, et al. Disease-free survival according to degree of HER2 amplification for patients treated with adjuvant chemotherapy with or without 1 year of trastuzumab: the HERA Trial. J Clin Oncol 2009;27:2962-9.

68. Osborne CK, Schiff R. Estrogen-receptor biology: continuing progress and therapeutic implications. J Clin Oncol 2005;23: 1616-22.

69. Xia L, Zhang D, Du R, et al. miR-15b and miR-16 modulate multidrug resistance by targeting BCL2 in human gastric cancer cells. Int J Cancer 2008;123:372-9.

70. Cittelly DM, Das PM, Salvo VA, et al. Oncogenic HER2\{Delta\}16 suppresses miR-15a/16 and deregulates BCL-2 to promote endocrine resistance of breast tumours. Carcinogenesis 2010;31:2049-57.

71. Cimmino A, Calin GA, Fabbri M, et al. miR15 and miR-16 induce apoptosis by targeting BCL2. Proc Natl Acad Sci USA 2005; 102:13944-9.

72. Finkle D, Quan ZR, Asghari V, et al. HER2 targeted therapy reduces incidence and progression of midlife mammary tumours in female murine mammary tumour virus huHER2-transgenic mice. Clin Cancer Res 2004;10:2499-511.

73. Christianson TA, Doherty JK, Lin YJ, et al. NH2-terminally truncated HER-2/neu protein: relationship with shedding of the extracellular domain and with prognostic factors in breast cancer. Cancer Res 1998;58:5123-9.

74. Yuan CX, Lasut AL, Wynn R, et al. Purification of Her-2 extracellular domain and identification of its cleavage site. Protein Expr Purif 2003;29:217-22.

75. Liu PC, Liu X, Li Y, et al. Identification of ADAM10 as a major source of HER2 ectodomain sheddase activity in HER2 overexpressing breast cancer cells. Cancer Biol Ther 2006;5:657-64.

76. Pedersen K, Angelini PD, Laos S, et al. A naturally occurring HER2 carboxy-terminal fragment promotes mammary tumour 
growth and metastasis. Mol Cell Biol 2009;29:3319-31.

77. Garcia-Castillo J, Pedersen K, Angelini PD, et al. HER2 carboxyl-terminal fragments regulate cell migration and cortactin phosphorylation. J Biol Chem 2009;284:2530213.

78. Molina MA, Saez R, Ramsey EE, et al. $\mathrm{NH}(2)$-terminal truncated HER-2 protein but not full-length receptor is associated with nodal metastasis in human breast cancer. Clin Cancer Res 2002;8:347-53.
79. Saez R, Molina MA, Ramsey EE, et al. p95HER-2 predicts worse outcome in patients with HER-2-positive breast cancer. Clin Cancer Res 2006;12:424-31.

80. Scaltriti M, Rojo F, Ocana A, et al. Expression of p95HER2, a truncated form of the HER2 receptor, and response to antiHER2 therapies in breast cancer. J Natl Cancer I 2007;99:628-38.

81. Sperinde J, Jin X, Banerjee J, et al. Quantitation of p95HER2 in paraffin sections by using a p95-specific antibody and correlation with outcome in a cohort of trastuzumab-treated breast cancer patients. Clin Cancer Res 2010;16:4226-35.

82. Scaltriti M, Chandarlapaty S, Prudkin L, et al. Clinical benefit of lapatinib-based therapy in patients with human epidermal growth factor receptor 2-positive breast tumours coexpressing the truncated p95HER2 receptor. Clin Cancer Res 2010; 16:2688-95. 
